\title{
Rotational mixing in low-mass stars
}

\section{Effect of the $\mu$-gradients in main sequence and subgiant Pop I stars}

\author{
A. Palacios ${ }^{1}$, S. Talon ${ }^{2}$, C. Charbonnel ${ }^{1}$, and M. Forestini ${ }^{3}$ \\ ${ }^{1}$ Laboratoire d'Astrophysique de Toulouse, CNRS UMR5572, OMP, 14 Av. E. Belin, 31400 Toulouse, France \\ 2 Département de Physique, Université de Montréal, Montréal PQ H3C 3J7, Canada; CERCA, 5160 Boul. Décarie, \\ Montréal PQ H3X 2H9, Canada \\ ${ }^{3}$ Laboratoire d'Astrophysique de l'Obs. de Grenoble, 414 rue de la Piscine, 38041 Grenoble Cedex 9, France
}

Received 22 August 2002 / Accepted 22 October 2002

\begin{abstract}
We present a first set of results concerning stellar evolution of rotating low-mass stars. Our models include fully consistent transport of angular momentum and chemicals due to the combined action of rotation induced mixing (according to Maeder \& Zahn 1998) and element segregation. The analysis of the effects of local variations of molecular weight due to the meridional circulation on the transport of angular momentum and chemicals are under the scope of this study. We apply this mechanism to low mass main sequence and subgiant stars of population I.

We show that the so-called $\mu$-currents are of major importance in setting the shape of the rotation profile, specially near the core. Furthermore, as shown by Talon \& Charbonnel (1998) and Charbonnel \& Talon (1999) using models without $\mu$-currents, we confirm that rotation-induced mixing in stars braked via magnetic torquing can explain the blue side of the Li dip, as well as the low $\mathrm{Li}$ abundances observed in subgiants even when $\mu$-currents are taken into account. We emphasize that $\mu$ variations are not to be neglected when treating rotation-induced mixing, and that they could be of great importance for latter evolutionary stages.
\end{abstract}

Key words. stars: interiors - stars: rotation - stars: abundances - hydrodynamics - turbulence

\section{Introduction}

Whereas standard stellar evolution models (allowing solely for mixing in convective regions) represent a good tool for a zeroth order description of stellar observations, they cannot account for series of abundance patterns observed in various locations of the Hertzsprung-Russell diagram.

While some abundance anomalies are best explained in terms of surface features (as is the case of Am stars for example; see Turcotte et al. 2000), others probably involve deep mixing (as the He overabundances in O stars; see Maeder \& Meynet 2000 for details). Rotation induced mixing, in the form of meridional circulation, baroclinic and shear instabilities, in conjunction with microscopic diffusion has been extensively used in order to reduce the discrepancies between standard models and observations, and has proved to be quite promising.

However, following rotation induced mixing in stellar evolution codes is challenging and, up to now, many of the theoretical results from numerical computations have assumed some hypothesis that could be too restrictive. Here, we wish to

Send offprint requests to: A. Palacios, e-mail: apalacio@ast.obs-mip.fr further examine the role of horizontal chemical inhomogeneities in the evolution of meridional circulation.

As first pointed out by Mestel (1953), meridional circulation is generated by a departure from spherical symmetry, and settles to restore it, but in turn generates itself a new dissymmetry: the meridian currents transport material with different mean molecular weights from one place to another of the radiative zone, so that a non-spherical $\mu$-distribution is set up which retroacts on the circulation, diminishing its magnitude. In this early description, assuming that the radiative zone does not suffer any local turbulent mixing and that the star remains in solid body rotation, this retroaction was actually shown to inhibit totally the circulation in chemically inhomogeneous regions.

Kippenhahn (1974) pointed out the importance of taking into account the changes in molecular weight, particularly because a strong $\nabla_{\mu}$ may represent a barrier that hinders mixing. In the models he proposes to explain the Am phenomenon, Vauclair (1977) emphasizes also the important role of the $\mu$-barrier and underlines the fact that it can considerably delay mixing below the convective envelope of slow rotators. This point, and more precisely the retroaction effect of the $\mu$-currents 
on meridional circulation still in the case of Am stars, was rediscussed further by Moss (1979).

This idea of the chocking of meridional circulation by mean molecular gradients was pushed further by Zahn (1992). In the formalism he develops the vertical velocity of meridional circulation, $U$, can be written as the sum of two major terms, one describing the effects of rotation in a homogeneous star, and the other accounting for the effects of chemical inhomogeneities on the circulation (see Sect. 2). In this description, he emphasizes the fact that, due to the strong vertical stratification in stars, horizontal motions are much favoured compared to vertical ones and that strongly anisotropic turbulence is likely to set in (such a description is also used by Tassoul \& Tassoul 1982). The effect of that turbulence is to mix chemicals horizontally, thus slowly destroying the horizontal inhomogeneities that give rise to $\mu$-currents.

Vauclair (1999) uses simplified expressions for rotational mixing and couples them to microscopic diffusion in order to examine their combined effect in slowly solid-body rotating stars. After the build-up of the horizontal $\mu$ gradients that block the $\Omega$-currents as in Mestel's description, she describes a selfregulated process which could freeze both meridional circulation and gravitational settling.

In the present paper, we present complete treatment of rotation induced mixing, including meridional circulation, turbulence by shear instabilities and microscopic diffusion. We will describe the effects of such a combination of processes on the transport of both angular momentum and chemical species in main sequence and subgiant Pop I stars. Concerning the later point, we refer to the papers of Talon \& Charbonnel (1998) and Charbonnel \& Talon (1999) (hereafter TC98 and CT99 respectively) who investigated these kind of processes under the scope of the so-called lithium dip problem, but did not include the effects of the variations of the mean molecular weight on the mixing.

We will only consider stars that, according to observations in open clusters, lie on the hot side of the lithium dip. Indeed, as pointed out by TC98, other transport processes for momentum are known to act in lower mass stars. In particular, the rotation induced mixing we implemented here fails to reproduce the solar rotation profile as inferred from helioseismology (Matias \& Zahn 1997), a conclusion also reached using other versions of detailed rotation induced mixing (Chaboyer et al. 1995). TC98 suggested that the additional mechanism responsible for momentum transport in the Sun could well explain the rise of $\mathrm{Li}$ abundances on the red side of the Li dip, and it is the point of view we adopt here by restricting our study to the blue side.

We intend to apply our description of rotational induced mixing to abundance anomalies in evolved stars (red giants) in a forthcoming study and will therefore set here the theoretical stage that we will use in this and in papers to come. In Sect. 2, we give a detailed description of the physics at work, recalling the basis of the theory presented in Zahn (1992) and Maeder \& Zahn (1998). We will then (Sect. 3) describe the numerical method as well as the inputs used in the models presented. In Sect. 4 we compare the models including mixing to standard evolutionary tracks, and analyze in more details the effects of the $\mu$-currents on the transport in Sect. 5. We will finally compare our results to observations of main sequence and subgiant stars in Sect. 6, before giving a summary and conclusions in Sect. 7.

\section{Rotation induced mixing}

Meridional circulation takes place in the radiative interiors of rotating stars where it is generated by the thermal imbalance induced by the departure from spherical symmetry (Eddington 1925; Vogt 1926). Once established, this large scale circulation generates in turn advection of momentum and thus favours the development of strong horizontal diffusion through shear instability, which is the main source of turbulence ${ }^{1}$ (Zahn 1992; Talon \& Zahn 1997; Maeder \& Zahn 1998). On the other hand, vertical turbulence is partly inhibited due to the high level of stratification in this direction.

Under these conditions of strong anisotropic turbulence, one can use the hypothesis of the so-called "shellular rotation" (Zahn 1992). In this configuration $\Omega \simeq \Omega(P)$ for differential rotation is weak on isobars. All quantities depend solely on pressure and can be split into a mean value and its latitudinal perturbation

$f(P, \theta)=\bar{f}(P)+\tilde{f}(P) P_{2}(\cos \theta)$

where $P_{2}(\cos \theta)$ is the Legendre polynomial of order two. The combined effects of meridional circulation and horizontal turbulence lead to the vertical transport of chemical species and angular momentum.

The transport of angular momentum obeys an advectiondiffusion equation treated in Lagrangian coordinates, namely

$\rho \frac{\mathrm{d}\left(r^{2} \Omega\right)}{\mathrm{d} t}=\frac{1}{5 r^{2}} \frac{\partial}{\partial r}\left(\rho r^{4} \Omega U r\right)+\frac{1}{r^{2}} \frac{\partial}{\partial r}\left(r^{4} \rho v_{\mathrm{v}} \frac{\partial \Omega}{\partial r}\right)$,

$r$ being the radius, $\rho$ the density and $v_{\mathrm{v}}$ the vertical component of the turbulent viscosity. The vertical component of the meridional velocity is given by

$$
\begin{aligned}
U(r)= & \frac{P}{C_{\mathrm{p}} \rho T g\left[\nabla_{\mathrm{ad}}-\nabla+\varphi / \delta \nabla_{\mu}\right]} \\
& \times\left[\frac{L}{M}\left(E_{\Omega}+E_{\mu}\right)+\frac{C_{p} T}{\delta} \frac{\partial \Theta}{\partial t}\right]
\end{aligned}
$$

where $L$ is the luminosity, $M$ the mass, $P$ the pressure, $C_{\mathrm{p}}$ the specific heat at constant pressure, $T$ the temperature, $\nabla_{\text {ad }}, \nabla$ and $\nabla_{\mu}$ the adiabatic, radiative and mean molecular weight gradients respectively (Maeder \& Zahn 1998). $g$ is the modulus of the effective gravity, defined by the hydrostatic equilibrium equation

$\boldsymbol{g}=\boldsymbol{\nabla} \Phi+\frac{1}{2} \Omega^{2} \nabla s^{2}$

${ }^{1}$ Baroclinic instabilities might set in as well, but we adopt the view that they will be less efficient than the shear. This point is discussed by Zahn (1992) and Talon \& Zahn (1997). 
$\varphi=-\xi_{\mu} / \xi_{\rho}$ and $\delta=\xi_{T} / \xi_{\rho}$, where $\xi_{\mu}, \xi_{\rho}$ and $\xi_{T}$ are the coefficients in the general expression of the equation of state

$$
\begin{aligned}
\mathrm{d} \ln P= & \underbrace{\left.\frac{\partial \ln P}{\partial \ln \rho}\right|_{T, \mu} \mathrm{d} \ln \rho}_{\xi_{\rho}} \\
& +\underbrace{\left.\frac{\partial \ln P}{\partial \ln T}\right|_{\rho, \mu}}_{\xi_{T}} \mathrm{~d} \ln T+\underbrace{\left.\frac{\partial \ln P}{\partial \ln \mu}\right|_{T, \rho}}_{\xi_{\mu}} \mathrm{d} \ln \mu .
\end{aligned}
$$

$E_{\Omega}$ and $E_{\mu}$ depend respectively on the rotation profile and on the mean molecular weight as

$$
\begin{aligned}
E_{\Omega}= & 2\left[1-\frac{\overline{\Omega^{2}}}{2 \pi G \bar{\rho}}-\frac{\left(\bar{\varepsilon}+\bar{\varepsilon}^{\text {grav }}\right)}{\varepsilon_{\mathrm{m}}}\right] \frac{\tilde{g}}{\bar{g}} \\
& -\frac{\rho_{\mathrm{m}}}{\bar{\rho}}\left\{\frac{r}{3} \frac{\mathrm{d}}{\mathrm{d} r}\left[H_{\mathrm{T}} \frac{\mathrm{d}}{\mathrm{d} r}\left(\frac{\Theta}{\delta}\right)-\chi_{\mathrm{T}}\left(\frac{\Theta}{\delta}\right)+\left(1-\frac{1}{\delta}\right) \Theta\right]\right. \\
& \left.-\frac{2 H_{\mathrm{T}}}{r}\left(1+\frac{D_{\mathrm{h}}}{K}\right)\left(\frac{\Theta}{\delta}\right)+\frac{2}{3} \Theta\right\} \\
& -\frac{\left(\bar{\varepsilon}+\bar{\varepsilon}^{\text {grav }}\right)}{\varepsilon_{\mathrm{m}}}\left[H_{\mathrm{T}} \frac{\mathrm{d}}{\mathrm{d} r}\left(\frac{\Theta}{\delta}\right)+\left(f_{\varepsilon} \varepsilon_{\mathrm{T}}-\chi_{\mathrm{T}}\right)\left(\frac{\Theta}{\delta}\right)\right. \\
& \left.+\left(2-f_{\varepsilon}-\frac{1}{\delta}\right) \Theta\right]
\end{aligned}
$$

and

$$
\begin{aligned}
E_{\mu}= & \frac{\rho_{\mathrm{m}}}{\bar{\rho}}\left\{\frac{r}{3} \frac{\mathrm{d}}{\mathrm{d} r}\left[H_{\mathrm{T}} \frac{\mathrm{d}}{\mathrm{d} r}\left(\frac{\varphi}{\delta} \Lambda\right)-\left(\chi_{\mu}+\frac{\varphi}{\delta} \chi_{\mathrm{T}}+\frac{\varphi}{\delta}\right) \Lambda\right]\right. \\
& \left.-\frac{2 H_{\mathrm{T}}}{r}\left(\frac{\varphi}{\delta} \Lambda\right)\right\} \\
& +\frac{\left(\bar{\varepsilon}+\bar{\varepsilon}^{\mathrm{grav}}\right)}{\varepsilon_{\mathrm{m}}}\left[H_{\mathrm{T}} \frac{\mathrm{d}}{\mathrm{d} r}\left(\frac{\varphi}{\delta} \Lambda\right)+f_{\varepsilon}\left(\varepsilon_{\mathrm{T}}+\frac{\varphi}{\delta} \varepsilon_{\mu}\right)-\chi_{\mu}\right. \\
& \left.-\frac{\varphi}{\delta}\left(\chi_{\mathrm{T}}+1\right) \Lambda\right]
\end{aligned}
$$

where $H_{\mathrm{T}}$ is the temperature scale height, $K$ the thermal diffusivity and $f_{\varepsilon} \equiv \bar{\varepsilon} /\left(\bar{\varepsilon}+\bar{\varepsilon}^{\text {grav }}\right)$, with $\bar{\varepsilon}$ and $\bar{\varepsilon}^{\text {grav }}$ being respectively the mean nuclear and gravitational energy.

$\chi_{\mu}$ and $\varepsilon_{\mu}$ are logarithmic derivatives of the radiative conductivity $\chi$ and the total energy $\varepsilon$ with respect to $\mu$, while derivatives with respect to T are noted as $\chi_{\mathrm{T}}$ and $\varepsilon_{\mathrm{T}}$.

$\varepsilon_{\mathrm{m}}(r) \equiv L(r) / M(r)$ and $\rho_{\mathrm{m}}$ is the mean density inside the considered level surface.

$D_{\mathrm{h}}$ is the horizontal diffusion coefficient (see Eq. (18)). $\Theta$ and $\Lambda$ are the relative variations over an isobar of density and mean molecular weight respectively, with

$\Theta=\frac{\tilde{\rho}}{\bar{\rho}}=\frac{r^{2}}{3 g} \frac{\mathrm{d} \Omega^{2}}{\mathrm{~d} r} \quad$ and $\quad \Lambda=\frac{\tilde{\mu}}{\mu}$.

We have considered non-stationarity, foreseeing papers to come where the mixing processes described here will be further investigated inside RGB stars. The effects of non-stationarity on the circulation, which need to be included as soon as nuclear burning takes place in shells, appear through the terms $\partial \Theta / \partial t$ and $\bar{\varepsilon}^{\text {grav }}$ (Maeder \& Zahn 1998).

As shown by Chaboyer \& Zahn (1992), the vertical transport of chemicals through the combined action of vertical advection and strong horizontal diffusion can be described as a pure diffusive process. Indeed, splitting the concentration $c_{i}$ of the $i$ th element according to Eq. (1), the advective transport in the rigorous evolution equation for the mean concentration

$$
\frac{\partial \overline{c_{i}}}{\partial t}+\frac{1}{r^{2}} \frac{\partial r^{2} \rho\left\langle\tilde{c_{i}} U\right\rangle}{\partial r}=\frac{1}{r^{2}} \frac{\partial}{\partial r}\left[r^{2} \rho D_{\mathrm{v}} \frac{\partial \overline{c_{i}}}{\partial r}\right]
$$

can be replaced by a diffusive term, with an effective diffusion coefficient given by

$D_{\text {eff }}=\frac{|r U(r)|^{2}}{30 D_{\mathrm{h}}}$.

The vertical transport of chemicals then obeys a diffusion equation which, in addition to this macroscopic transport, also accounts for (vertical) turbulent transport, microscopic diffusion (see Sect. 3) and nuclear reactions:

$$
\begin{aligned}
\rho \frac{\mathrm{d} \overline{c_{i}}}{\mathrm{~d} t}= & \dot{c}_{i}+\frac{1}{r^{2}} \frac{\partial}{\partial r}\left[r^{2} \rho U_{\mathrm{diff}} \overline{c_{i}}\right] \\
& +\frac{1}{r^{2}} \frac{\partial}{\partial r}\left[r^{2} \rho\left(D_{\mathrm{eff}}+D_{v}\right) \frac{\partial \overline{c_{i}}}{\partial r}\right] .
\end{aligned}
$$

Microscopic diffusion appears through the velocity term $\left(\propto U_{\text {diff }}\right)$, and $\dot{c}_{i}$ represents the variations of chemical composition due to nuclear reactions. $D_{\mathrm{v}}$ is the vertical turbulent diffusion coefficient, taken equal to the turbulent viscosity (Zahn 1992). The total diffusion coefficient $D_{\text {tot }}$ for chemicals can be written as the sum of three coefficients:

$D_{\text {tot }}=D_{\text {mic }}+D_{\text {eff }}+D_{\mathrm{v}}$.

$\Lambda$ is tightly related to the horizontal inhomogeneities of chemicals, and obeys a similar law as the concentration of the chemicals under the assumption of shellular rotation,

$\frac{\partial \Lambda}{\partial t}+U \frac{\partial \ln \bar{\mu}}{\partial r}=-\frac{6}{r^{2}} D_{\mathrm{h}} \Lambda$

(see Chaboyer \& Zahn 1992 and Maeder \& Zahn 1998 for details). Even though this equation admits a stationary solution for $t \gg r^{2} / 6 D_{h}$, we will follow its time evolution since it allows for greater stability in the numerical algorithms.

Because of the low viscosity in stellar interiors, vertical shear due to differential rotation eventually becomes turbulent, and we will assume that this instability dominates, noting that baroclinic instabilities may also grow. However, development of the shear instability is possible only under certain conditions which are fulfilled when the flow satisfies to both the Reynolds and the Richardson instability criteria.

The Richardson criterion compares the stabilizing effect of the entropy gradient to the amount of energy that can be extracted from differential rotation. The instability condition is in general written as

$R i=\frac{N^{2}}{(\mathrm{~d} u / \mathrm{d} z)^{2}} \leq R i_{\text {crit }} \simeq \frac{1}{4}$.

This criterion is extremely severe and is seldom satisfied in stellar interiors.

However, as first pointed out by Townsend (1958), thermal diffusivity can decrease the stabilizing effect of entropy stratification. Maeder (1995) used this characteristic to show that 
in actual conditions, there will always exist a small enough length scale that will make the fluid unstable. The modified Richardson criterion becomes

$R i=\frac{\left(\frac{\Gamma}{\Gamma+1}\right) N_{\mathrm{T}}^{2}+N_{\mu}^{2}}{(\mathrm{~d} u / \mathrm{d} z)^{2}} \leq R i_{\text {crit }}$

where $\Gamma=v \ell / 6 K, v$ is the turbulent velocity, $\ell$ the turbulent length scale, $K$ is the thermal diffusivity, $N_{\mathrm{T}}^{2}$ is the thermal part of the Brünt-Väisälä frequency and $N_{\mu}^{2}$ is related to mean molecular weight gradients. $\Gamma$ can be viewed as a turbulent Peclet number, the turbulent viscosity $v_{\mathrm{v}}=v \ell$ replacing the normal microscopic viscosity. The turbulent viscosity is then obtained by setting the equality in Eq. (15). Further discussion on these topics may be found in Canuto (1998), who suggests that, in such a context, the critical Richardson number might actually be closer to unity.

In order for the instability to grow, the associated turbulent viscosity must also be larger than the microscopic viscosity $v$, as expressed by the Reynolds criterion

$v_{\mathrm{v}} \leq v R e_{\mathrm{c}}$

where $R e_{\mathrm{c}}$ is the critical Reynolds number $(\simeq 10)$.

The estimation of the appropriate formulation for the Richardson criterion is critical to give a good estimation of the turbulent viscosity, and in turn of the turbulent diffusion coefficient. Meynet \& Maeder (2000) present a critical review of the different expressions proposed so far concerning this coefficient. In the present work, we shall take the expression derived by Talon \& Zahn (1997) for the vertical component of the turbulent viscosity

$D_{\mathrm{v}}=v_{\mathrm{v}}=\frac{8}{5} \frac{R i_{\text {crit }}(r \mathrm{~d} \Omega / \mathrm{d} r)^{2}}{N_{\mathrm{T}}^{2} /\left(K+D_{\mathrm{h}}\right)+N_{\mu}^{2} / D_{\mathrm{h}}}$,

which adds to Maeder's prescription by also taking into account the weakening effect of the strong horizontal diffusion on the entropy stratification associated with the (vertical) $\mu$ gradients. The factor $\frac{8}{5}$ includes, in addition to a geometrical factor, the coefficient $\frac{2}{5}$ found by Maeder (1995) when deriving the criterion for shear instabilities assuming spherical geometry for the turbulent eddies.

$D_{\mathrm{h}}$ is the horizontal diffusion coefficient and is such as $D_{\mathrm{h}} \gg D_{\mathrm{v}}$, thus leading to strong anisotropic turbulence, which is one of the basic hypothesis of the derivation presented. This coefficient appears directly in Eq. (13) describing the evolution of the variations of $\Lambda$, as well as in the vertical and effective diffusivity coefficients. Its evaluation is the weakest point of the theory first developed by Zahn (1992). In the present paper, we use an expression linking this coefficient to the advection of angular momentum

$D_{\mathrm{h}}=\frac{r}{C_{\mathrm{h}}}\left|\frac{1}{3 \rho r} \frac{\mathrm{d}\left(\rho r^{2} U\right)}{\mathrm{d} r}-\frac{U}{2} \frac{\mathrm{d} \ln r^{2} \Omega}{\mathrm{d} \ln r}\right|$.

$C_{\mathrm{h}}$ is a free parameter of order unity (see Zahn 1992) which describes the weakening effect of horizontal turbulence on the vertical transport of chemicals. It appears through $D_{\mathrm{h}}$ in both effective $\left(D_{\text {eff }}\right)$ and turbulent $\left(D_{\mathrm{v}}\right)$ diffusion coefficients as can be seen from Eqs. (10) and (17). As $D_{\text {eff }} \propto C_{\mathrm{h}}$ and $D_{\mathrm{v}} \propto C_{\mathrm{h}}{ }^{-1}$, the net effect of varying this parameter on the global diffusion coefficient $D_{\text {tot }}$ might not be very important.

On the other hand, the magnitude of turbulent diffusion $D_{\mathrm{v}}$ depends on $R i_{\text {crit }}$ (see Eq. (17)). Changing the Richardson criterion as previously suggested, by using a larger critical number $R i_{\text {crit }}$, will result in an enhancement of $D_{\mathrm{v}}$ and in turn of the global diffusion coefficient.

\section{Numerical simulations: inputs}

We present and discuss models computed with the Grenoble stellar evolution code, STAREVOL. We included the transport of angular momentum and chemical species due to the combined action of meridional circulation, turbulence and microscopic diffusion (in the case of chemicals), following the formalism of Maeder \& Zahn (1998) as described in the previous section.

\subsection{Numerical treatment of the transport of angular momentum}

The 4th order differential equation for the transport of angular momentum (Eq. (2)) is split into four 1st order equations. The system is complemented by Eq. (13) in order to follow the feedback of variations of the mean molecular weight on transport.

We use the Newton-Raphson method according to Henyey (1964) to solve for angular momentum transport within the evolutionary code. The first boundary conditions impose momentum conservation at convective boundaries

$$
\begin{array}{ll}
\frac{\partial}{\partial t}\left[\Omega \int_{r_{\mathrm{t}}}^{R} r^{4} \rho \mathrm{d} r\right]=-\frac{1}{5} r^{4} \rho \Omega U+\mathcal{F}_{\Omega} & \text { for } r=r_{\mathrm{t}} \\
\frac{\partial}{\partial t}\left[\Omega \int_{0}^{r_{\mathrm{b}}} r^{4} \rho \mathrm{d} r\right]=\frac{1}{5} r^{4} \rho \Omega U & \text { for } r=r_{\mathrm{b}}
\end{array}
$$

We complement them by requiring the absence of differential rotation at convective boundaries

$$
\frac{\partial \Omega}{\partial r}=0 \quad \text { for } r=r_{\mathrm{t}}, r_{\mathrm{b}}
$$

We also impose $\Lambda=0$ there, a natural consequence of the fact that convective regions are assumed to be chemically homogeneous. $r_{\mathrm{t}}$ and $r_{\mathrm{b}}$ correspond to the top (surface) and bottom (center) of the radiative zone respectively. $\mathcal{F}_{\Omega}$ represents the torque applied at the surface of the star. Note that momentum conservation is equivalent to $U=0$ when there is no convective core.

In the models presented here, we considered a classical perfect gas so that $\delta=\varphi=1$ in Eqs. (3), (5) and (7). We also used $C_{\mathrm{h}}=1$ and $R i_{\mathrm{c}}=0.25$, thus not allowing these two parameters to vary.

The transport of chemicals according to Eq. (11) is treated via a finite element method. Boundary conditions impose 
conservation of the integrated mass of each element and can be written as follows

$$
\begin{aligned}
& \frac{\partial}{\partial t}\left[c_{i} \int_{r_{\mathrm{t}}}^{R} r^{2} \rho \mathrm{d} r\right]=-r^{2} \rho\left(U_{\mathrm{diff}} \overline{c_{i}}\right)-r^{2} \rho\left(D_{\mathrm{v}}+D_{\mathrm{eff}}\right) \frac{\partial c_{i}}{\partial r} \\
& -\dot{M} c_{i} \quad \text { for } r=r_{\mathrm{t}} \\
& \begin{array}{r}
\frac{\partial}{\partial t}\left[c_{i} \int_{0}^{r_{\mathrm{b}}} r^{2} \rho \mathrm{d} r\right]=r^{2} \rho\left(U_{\mathrm{diff}} \overline{c_{i}}\right)+r^{2} \rho\left(D_{\mathrm{v}}+D_{\mathrm{eff}}\right) \frac{\partial c_{i}}{\partial r} \\
\text { for } r=r_{\mathrm{b}},
\end{array}
\end{aligned}
$$

where $\dot{M}$ is the mass loss rate.

We emphasize that rotational induced mixing is treated in a self-consistent way and that coupling to the evolutionary code ensures feedback of transport mechanisms on structure at all evolutionary phases.

\subsection{Structure equations}

One of the basic effects of rotation on internal structure is to break the spherical symmetry of the star. Rigorously, corrections due to this departure from spherical symmetry should thus be applied when writing the internal structure equations. Meynet \& Maeder (1997) developed a formalism suitable in the "shellular rotation" context. They showed that rotation has little influence on the hydrostatic structure of massive stars, while the effects at the stellar surface (in particular on the effective temperature) may be important. In the HR diagram, these effects may be of the same order of magnitude as those due to mixing. In the present work, we made the assumption of negligible departures from spherical symmetry, and we did not include these corrections (i.e., stellar structure equations are solved in a standard way). The effects on surface parameters such as $L$ and $T_{\text {eff }}$ discussed here are thus only due to rotationinduced mixing.

\subsection{Evolution of the rotational surface velocity}

As already mentioned, we only consider stars hotter than $6500 \mathrm{~K}$ on the ZAMS, associated to the hot side of the lithium dip in open clusters. We refer to the work of Gaigé (1993) on the Hyades F-stars to choose the initial velocity of our models and further determine the braking to be applied according to the temperature. For further discussion on rotation in F-stars, the reader is referred to TC98 and CT99 where these aspects are more extensively discussed.

In the present paper, stars have an initial velocity of $110 \mathrm{~km} \mathrm{~s}^{-1}$ and are assumed to undergo magnetic braking while arriving on main sequence (see Table 2) as suggested by the early work of Schatzman (1962). This braking was proved to be more efficient for thicker convective envelopes, that is to say for lower masses at given metallicity, a trend which is confirmed by the observations. Their rotational velocity is further reduced to only a few $\mathrm{km} \mathrm{s}^{-1}$ when they are on the subgiant branch due to the star's expansion.
The braking law adopted follows the description of Kawaler (1988)

$$
\frac{\mathrm{d} J}{\mathrm{~d} t}= \begin{cases}-K \Omega^{3}\left(\frac{R}{R_{\odot}}\right)^{1 / 2}\left(\frac{M}{M_{\odot}}\right)^{-1 / 2} & \left(\Omega \leq \Omega_{\mathrm{sat}}\right) \\ -K \Omega \Omega^{2}{ }_{\mathrm{sat}}\left(\frac{R}{R_{\odot}}\right)^{1 / 2}\left(\frac{M}{M_{\odot}}\right)^{-1 / 2} & \left(\Omega>\Omega_{\mathrm{sat}}\right) .\end{cases}
$$

This formulation corresponds to a field geometry intermediate between a dipolar and a radial field (Kawaler 1988). Neglecting the evolution of stellar structure and assuming solid body rotation, this leads to a Skumanich law in $t^{-1 / 2}$ for the surface velocity. It is widely used in the literature (Chaboyer et al. 1995; Krishnamurti et al. 1997; Bouvier et al. 1997; Sills \& Pinsonneault 2000). The parameter $\Omega_{\text {sat }}$ expresses the fact that magnetic field generation saturates at some critical value, as shown by various diagnostics of magnetic activity (see Saar 1996 and references therein). This saturation is actually required in order to retain a sufficient amount of fast rotators in young clusters, as originally suggested by Stauffer \& Hartmann (1987). The constant $K$ is related to the magnitude of the magnetic field strength. In particular, in the mass range we are studying here, the convection zone shrinks significantly from the $1.35 M_{\odot}$ to the $1.8 M_{\odot}$ model, and this parameter, which is normally calibrated on the Sun and taken to be a constant in all stars (see Bouvier et al. 1997) could very well vary here.

In studies of stars less massive than $\sim 1.2 M_{\odot}$, there is a suggestion that $\Omega_{\text {sat }}$ varies with mass, and a scaling in $\tau_{\text {conv }}^{-1}$ is invoked (Barnes \& Sofia 1996; Krishnamurti et al. 1997; Bouvier et al. 1997; Sills \& Pinsonneault 2000). This is required to explain the fact that $\mathrm{K}$ stars rotate more rapidly than $\mathrm{G}$ stars in the Hyades. However, any diagnosis depends on the actual model for momentum distribution in the stellar interior. For the masses under the scope of this study, braking remains much smaller than it is for lower mass stars, as reflected by the fact that at the age of the Hyades, even the less massive model is still rotating at $30 \mathrm{~km} \mathrm{~s}^{-1}$.

Table 1 presents the rotational characteristics of our models, as well as the global convective time-scale as defined by Kim \& Demarque (1996)

$\tau_{\text {conv }}=\int_{r_{\mathrm{t}}}^{R} \mathrm{~d} r / v_{\text {conv }}$.

The scaling of $K \Omega_{\text {sat }}^{2}$ is clearly not inversely proportional to $\tau_{\text {conv }}$. Furthermore, braking for stars more massive than $\sim 1.35 M_{\odot}$ is less efficient than it is in lower mass stars, while their convective time-scales are lower. We are thus lead to believe it is a mass range for which the value of $K$ itself varies, in agreement with our knowledge that it is also a transition region for stellar activity.

Let us note that all our calculations were performed in the saturated regime, and that our stars follow the evolution of the mean velocity for the 3 considered clusters ( $\alpha$ Per, Pleiades, Hyades). It is thus not possible to disentangle the effect of a variation of $\Omega_{\text {sat }}$ and a variation of $K$. 
Table 1. Rotational parameters of the models.

\begin{tabular}{cccccc}
\hline \hline $\begin{array}{c}M \\
\left(M_{\odot}\right)\end{array}$ & $K \Omega_{\text {sat }}^{2}$ & $\begin{array}{c}\tau_{\text {conv }} \\
\text { days }\end{array}$ & $\begin{array}{c}T_{\text {eff }} \\
(\mathrm{K})\end{array}$ & $\begin{array}{c}v \\
\left(\mathrm{~km} \mathrm{~s}^{-1}\right)\end{array}$ & Age \\
\hline 1.35 & $2.3 \times 10^{43}$ & 6.0 & 6620 & 70.3 & $\alpha$ Per \\
& & & 6630 & 43.6 & Pleiades \\
& & & 6625 & 30.6 & Hyades \\
1.4 & $4.4 \times 10^{42}$ & 4.0 & 6770 & 97.5 & $\alpha$ Per \\
& & & 6760 & 66.2 & Pleiades \\
& & & 6745 & 46.3 & Hyades \\
1.5 & $5.1 \times 10^{41}$ & \multirow{2}{*}{3.4} & 7080 & 100 & $\alpha$ Per \\
& & & 7045 & 83.0 & Pleiades \\
& & & 6990 & 76.0 & Hyades \\
1.8 & $7.1 \times 10^{40}$ & 0.44 & 8280 & 110 & $\alpha$ Per \\
& & & 8020 & 94.4 & Pleiades \\
& & & 7625 & 84.1 & Hyades \\
\hline
\end{tabular}

\subsection{Treatment of the microscopic diffusion}

We included microscopic diffusion in the form of gravitational settling as well as that related to thermal gradients, using the formulation of Paquette et al. (1986). Atomic diffusion for trace elements $\mathrm{Li}, \mathrm{Be}, \mathrm{B}$ must be addressed with care in the limited range of effective temperature on the left side of the Li dip. Indeed, Richer \& Michaud (1993) showed that, for main sequence stars with an effective temperature in the range $\sim 6900-7100 \mathrm{~K}$, radiative forces on $\mathrm{Li}$ and Be could lead to increased surface abundances, at least if large scale mixing is not efficient enough to counter balance them. The recent discovery of a slowly rotating Li rich dwarf (Deliyannis et al. 2002) is nicely explained in this framework. This had lead TC98 and CT99 to remove microscopic diffusion on those elements, in the corresponding temperature range. Here, we adopt a different view point. We compute a first series of models including microscopic diffusion (but not radiative forces) on all light elements. Then, for stars in the range of effective temperature between $\sim 6900-7100 \mathrm{~K}$, we also compute models without microscopic diffusion on $\mathrm{Li}, \mathrm{Be}$ and $\mathrm{B}$. The differences between these two series of models give an indication of the amount of microscopic diffusion that remains in our rotating models. As we shall show, this amount is minute. One must keep in mind though that the real effect of microscopic diffusion in these stars will be to slightly enhance $\mathrm{Li}$ and Be abundances rather than diminish them.

Let us note that radiative forces have a magnitude which is similar to that of settling (see Richer et al. 2000, Figs. 5 and 6). Thus, if mixing is efficient enough to (almost) inhibit settling, it will also inhibit levitation by radiation. However, some $\mathrm{Li}$ and/or Be overabundances could survive in the small $T_{\text {eff }}$ window where radiative forces are much larger.

For main sequence stars with $T_{\text {eff }}>7100 \mathrm{~K}$, radiative forces do not play a major role on $\mathrm{Li}$ and $\mathrm{Be}$ compared to atomic diffusion, which is thus the only process (apart from rotation-induced mixing) which is applied.

\subsection{Input physics}

A rather detailed description of our stellar evolution code can be found in Siess et al. (1997) (see also Siess et al. 2000 for more recent aspects). As far as main sequence and subgiant models are concerned, let us briefly summarize here the relevant physics. Our equation of state has been developed from the Pols et al. (1995) formalism. Thermodynamical features of each plasma component (ions, electrons and photons, as well as $\mathrm{H}^{-}$and $\mathrm{H}_{2}$ ) are obtained by minimizing the Helmholtz free energy that includes separately non-ideal effects (Coulomb shielding and pressure ionisation). In input, instead of the usual pressure or density variable, it uses a new independent variable that describes the electron degeneracy. This allows in turn to treat analytically the ionisation process and provides very smooth profiles of thermodynamical quantities. It is particularly well suited to treat partially ionised and partially degenerated regions, pressure ionisation and Coulomb interactions in a stellar evolution code. More details about this equation of state are given in Siess et al. (2000).

Radiative opacities are interpolated from Alexander \& Fergusson (1994) at temperatures below $8000 \mathrm{~K}$, and from Iglesias \& Rogers (1996) at higher temperatures. Atmospheres are integrated in the gray, plan-parallel and Eddington approximations. Our nuclear reaction network allows to follow the abundance evolution of 53 species (from ${ }^{1} \mathrm{H}$ to ${ }^{37} \mathrm{Cl}$ ) through 180 reactions. All nuclear reaction rates have been updated with the NACRE compilation (Angulo et al. 1999). The value of $\alpha_{\mathrm{p}}=l / H_{\mathrm{p}}$, the ratio of the mixing length to the pressure scale height, is taken equal to 1.75 . No overshooting is considered for convection.

\section{Global characteristics of the models}

We present evolutionary results for five different masses (1.35, $1.4,1.5,1.8$ and $2.2 M_{\odot}$ ) at $Z=0.02$. Models include complete treatment of angular momentum and chemicals transport, taking into account the effects of changes of $\mu\left(E_{\mu} \neq 0\right)$ and applying microscopic diffusion to all elements.

These models sample the effective temperature domain covered by the observations of main-sequence stars on the hot side of the lithium dip in young open clusters with nearly solar metallicity such as the Hyades, Coma Berenices and Praesepe. They also span the mass range of Pop I subgiants to which our results will be compared in a further section (Sect. 6).

In Fig. 1, we plot the evolutionary tracks of our rotating models in the Hertzsprung-Russell diagram (solid lines), comparing them to their standard analogs (dashed lines).

Table 2 presents some characteristics of the computed models with and without rotation. All models were computed with the same set of parameters for rotation induced mixing $\left(C_{\mathrm{h}}=1\right.$ and $R i_{\text {crit }}=0.25$ ), but with different rotational histories (see Sect. 3.2).

One can see clearly how mixing affects main-sequence evolution. In rotating models, the turn-off is moved towards cooler temperatures while the luminosity is slightly enhanced.

Due to surface effects, still cooler effective temperatures (by up to $\sim 100 \mathrm{~K}$ in the fastest stars considered here) would be 
Table 2. Characteristics of the stellar models and rotation velocities. Models computed with $Z=0.02$. The turn-off (TO) is defined as the point for which the central hydrogen mass fraction is lower than $10^{-9}$.

\begin{tabular}{cccccc}
\hline \hline $\begin{array}{c}M \\
\left(M_{\odot}\right)\end{array}$ & $\begin{array}{c}v_{\text {init }} \\
\left(\mathrm{km} \mathrm{s}^{-1}\right)\end{array}$ & $\begin{array}{c}v_{\text {Hyades }} \\
\left(\mathrm{km} \mathrm{s}^{-1}\right)\end{array}$ & $\begin{array}{c}T_{\text {eff, TO }} \\
(\mathrm{K})\end{array}$ & $\begin{array}{c}L_{\mathrm{TO}} \\
\left(L_{\odot}\right)\end{array}$ & $\begin{array}{c}t_{\mathrm{TO}} \\
(\mathrm{Gyrs})\end{array}$ \\
\hline 1.35 & 110 & 30 & 6113 & 5.92 & 4.140 \\
& 0 & 0 & 6328 & 5.68 & 3.418 \\
1.4 & 110 & 51 & 6218 & 6.92 & 3.555 \\
& 0 & 0 & 6451 & 6.42 & 2.885 \\
1.5 & 110 & 80 & 6382 & 8.80 & 2.967 \\
& 0 & 0 & 6585 & 8.38 & 2.404 \\
1.8 & 110 & 82 & 7001 & 17.81 & 1.470 \\
& 0 & 0 & 7255 & 17.17 & 1.317 \\
2.2 & 110 & 77 & 8034 & 39.41 & 0.788 \\
& 0 & 0 & 8228 & 39 & 0.746 \\
\hline
\end{tabular}

obtained if the effects of rotation on structure equations were taken into account (Peréz Hernández et al. 1999). Let us note that the actual value depends on the inclination angle of the star.

Our models are computed without convective core overshooting. We checked however that for the $1.8 M_{\odot}$ model, the effect of mixing on main sequence lifetime and on the turnoff in the HRD is equivalent to an overshooting distance for convection of about $0.1 H_{\mathrm{P}}$ (where $H_{\mathrm{P}}$ is the pressure scale height at the edge of the classical core). This value is smaller than the one (0.2) typically used to fit the observed color-magnitude diagrams of young and intermediate age galactic open clusters (Meynet et al. 1993; Lebreton 2000 and references therein). Whether the rotating models would fit the cluster isochrones remains to be checked. This requires a more extended grid of masses and is out of the scope of the present paper.

On the subgiant branch, stars evolve at higher luminosities. This is due to the effect of microscopic diffusion during the main sequence phase. Indeed, even if it is partly balanced by the action of large scale mixing, microscopic diffusion leads to the build up of a negative helium gradient at the base of the convective envelope. When the first dredge-up begins, the envelope encounters regions with a lower opacity (due to enhancement of helium) leading to enhanced luminosities compared to standard models. When reaching the RGB, differences in effective temperature and luminosity between standard and rotating models become marginal: both tracks are almost identical in the HR diagram (see Fig. 1).

Models with rotational mixing behave as if their metallicity was lower than in the standard cases. This trend was already emphasized by CT99.

On the other hand, the lifetimes of rotating models are enhanced with respect to those of standard models. In fact, due to the positive slope of the hydrogen profile, mixing slightly feeds the core with fresh hydrogen fuel. The exhaustion of hydrogen in the central region is delayed and the time spent on main sequence increases.

In the mass range we are studying here, the less massive the star, the more turn-off ages differ between rotating and

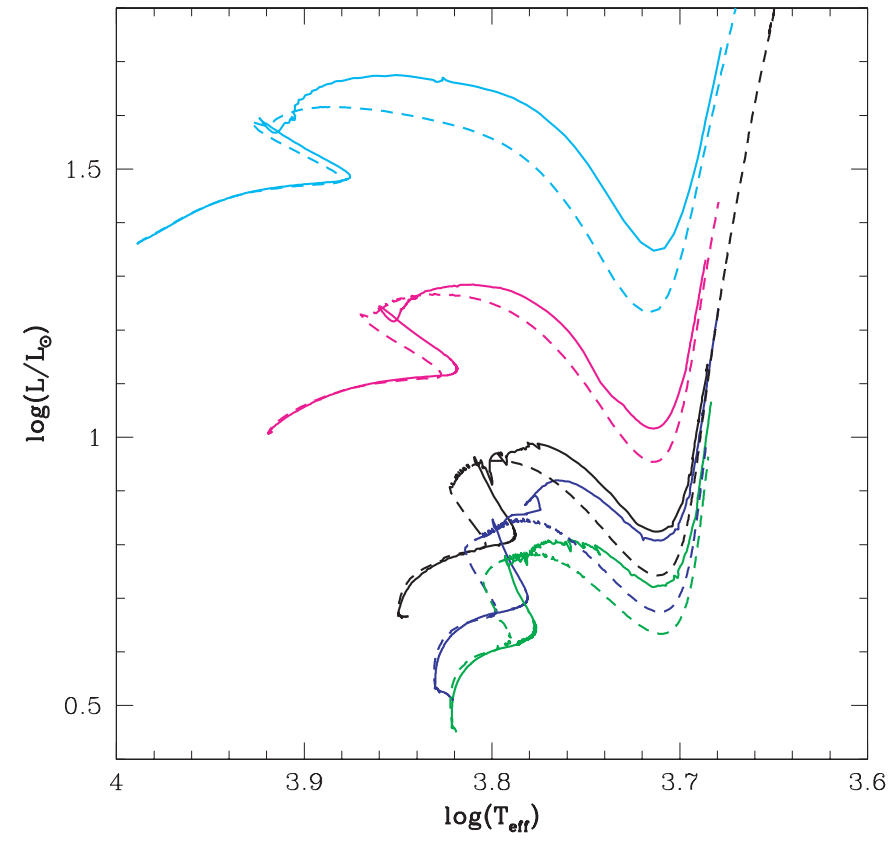

Fig. 1. HR diagrams for the five masses considered in this paper. Dashed lines are for standard models and solid lines are for models with the "complete mixing" and $C_{\mathrm{h}}=1 . Z=0.02$ for all models presented.

non-rotating models ${ }^{2}$. This trend with mass has to be related to braking, which is more important for lower mass stars as imposed by both theory and observations. Stronger mixing corresponds to a more efficient extraction of angular momentum at the surface, thus leading to enhanced meridional circulation and mixing. There should thus be a correlation between mixing and braking, as we will show from the comparison of our models with observations (see Sect. 6).

\section{Effects of $\nabla_{\mu}$ and $\mu$-currents}

To discuss the effects of chemical composition variations on transport, we will focus on the $1.5 M_{\odot}$ model. We present a comparative study of the results obtained with and without $\mu$ currents $\left(E_{\mu}=0\right.$ or $\left.E_{\mu} \neq 0\right)$. In both cases all parameters are kept identical in order to make the comparison clearer. Furthermore, we applied a torque so that surface velocity at the age of the Hyades $(\simeq 700 \mathrm{Myrs})$ is the same whether $E_{\mu}$ is zero or not.

\subsection{Meridional circulation velocity $U(r)$}

Figure 2 presents profiles of $U, \Omega$ and the diffusion coefficient $D_{\text {tot }}$ at different times on the main sequence, indicated in terms of central hydrogen mass fraction $\mathrm{X}_{\mathrm{c}}$. We superimpose the profiles obtained for $E_{\mu}=0$ (dashed lines) and $E_{\mu} \neq 0$ (solid lines).

When neglecting $\mu$-currents $\left(E_{\mu}=0\right)$, the meridional velocity is negative in the entire mixing zone. There is only one

\footnotetext{
2 The time spent on the main sequence is increased by more than $20 \%$ in the lower mass stars, and by $\approx 10 \%$ in the higher mass models.
} 

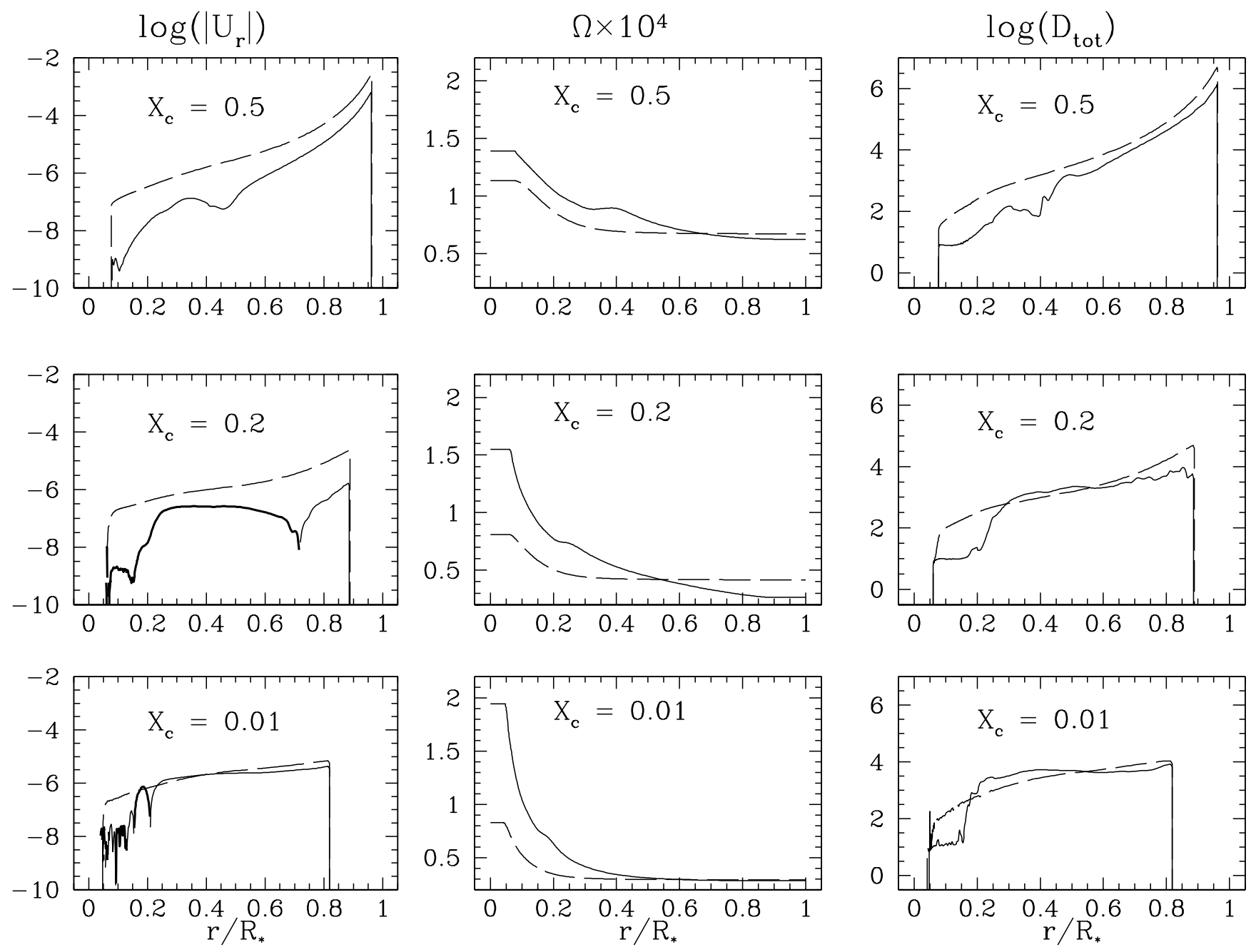

Fig. 2. Profiles of the vertical component of the meridional velocity $U_{\mathrm{r}}$ (left panels), angular velocity $\Omega$ (middle panels) and diffusion coefficients (right panels) inside a $1.5 M_{\odot}, Z=0.02$ star. Solid lines are for the model with $E_{\mu} \neq 0$ and dashed lines for the model with $E_{\mu}=0$. Central hydrogen content is as indicated. In the left panels, bold parts in the solid lines indicate positive values of $U_{\mathrm{r}}$.

meridian loop that brings matter upwards at the equator and down in polar regions. This leads to the extraction of angular momentum in the radiative zone, a consequence of braking. The amplitude of meridional velocity decreases as the star evolves and braking slows down.

When $E_{\mu}$ is not zero, some changes appear. There are now two loops of circulation. In the outer parts of the radiative zone, the negative loop keeps on transporting material from the equator up along the pole, in response to the extraction of angular momentum. In the interior however, circulation is positive, revealing an inward transport of angular momentum. Equations (3), (5) and (7) already suggest a strong interaction between $\Omega$ - and $\mu$-currents. Apart from the first term in Eq. (3), which includes Eddington-Sweet and Gratton-Öpik terms, $E_{\Omega}$ and $E_{\mu}$ are almost mirrored. At the beginning of the evolution, $\left|E_{\Omega}\right|>\left|E_{\mu}\right|$, for $\mu$-currents have not settled yet. As the star evolves and $\mu$-gradients built-up, $\mu$-currents may grow. $\left|E_{\mu}\right|$ and $\left|E_{\Omega}\right|$ eventually become of the same order of magnitude, and $U$ disminishes.

Contrary to what was expected by Mestel (1953) the circulation is not frozen. Indeed, the two terms $E_{\mu}$ and $E_{\Omega}$, while being of the same order, never compensate each other as can be seen in Fig. 3, where $U$ becomes very small, but never disappears completely. Indeed, due to the strength of the horizontal diffusion, the circulation must remain to continuously refresh the horizontal $\mu$ fluctuation. However, the difference between both contributions $E_{\Omega}$ and $E_{\mu}$ decreases significantly as the star evolves. Meridional circulation velocity is thus quite slow near the end of the main sequence, but transport of chemicals still occurs, as can be seen from the diffusion coefficient in Fig. 2.

\subsection{Rotation profile $\Omega$}

The inner transport of angular momentum also affects the rotation profiles (Fig. 2 middle panels): when including $\mu$-currents, differential rotation grows larger. In particular, the core gains momentum and gets accelerated as evolution proceeds instead of keeping a nearly constant rotation rate. This can be of crucial importance when further following the evolution of the star on the red giant branch. This behaviour may be interpreted as follows: the rate of differential rotation and the relative variations of mean molecular weight are related to each other via 

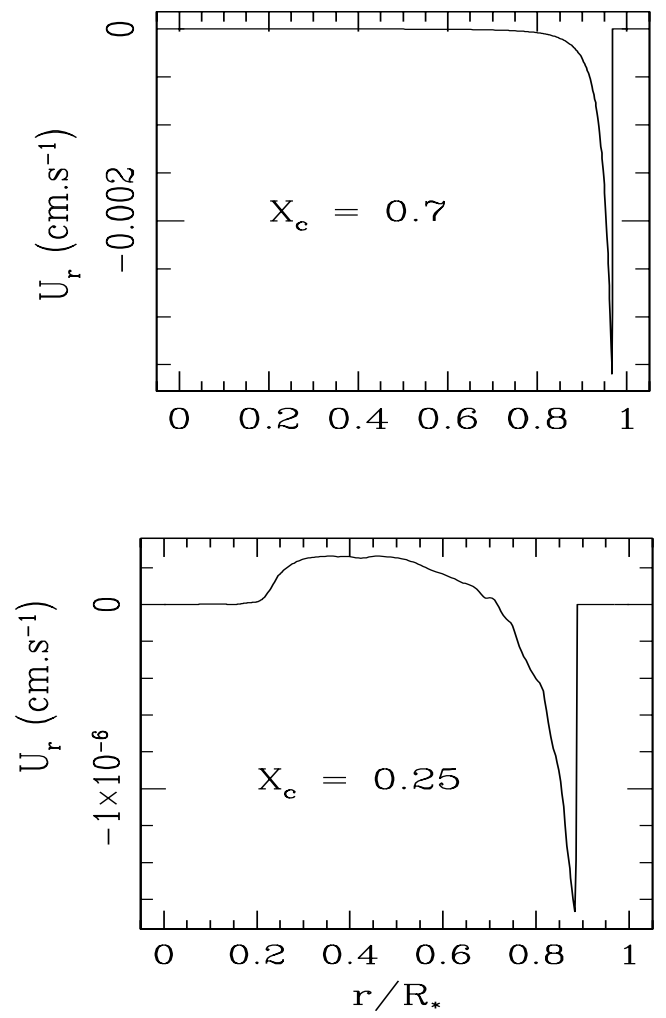
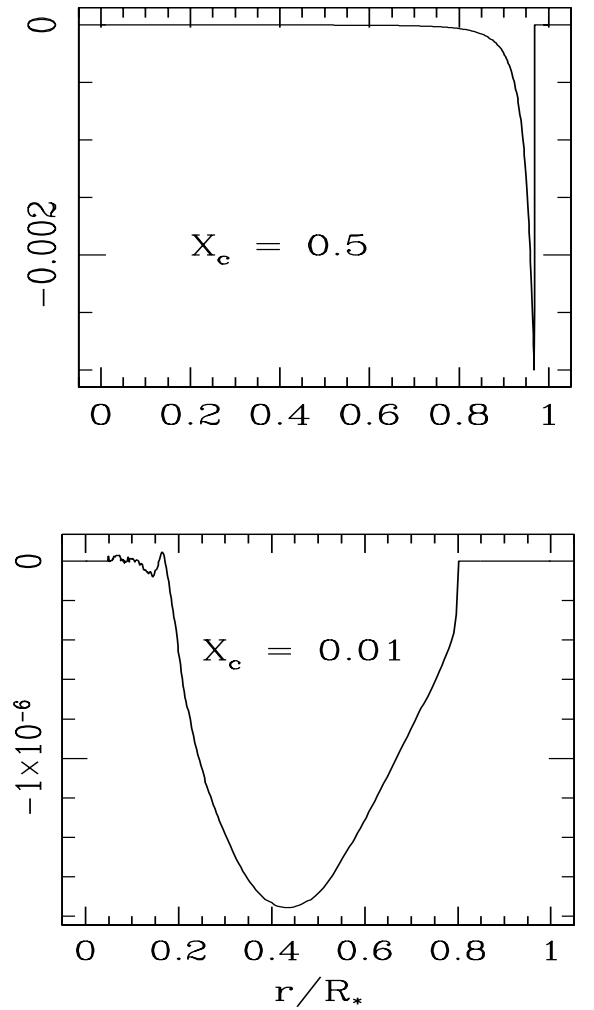

Fig. 3. Vertical component of the meridional circulation velocity, $U$ as a function of the reduced radius at the same epochs inside the same star.

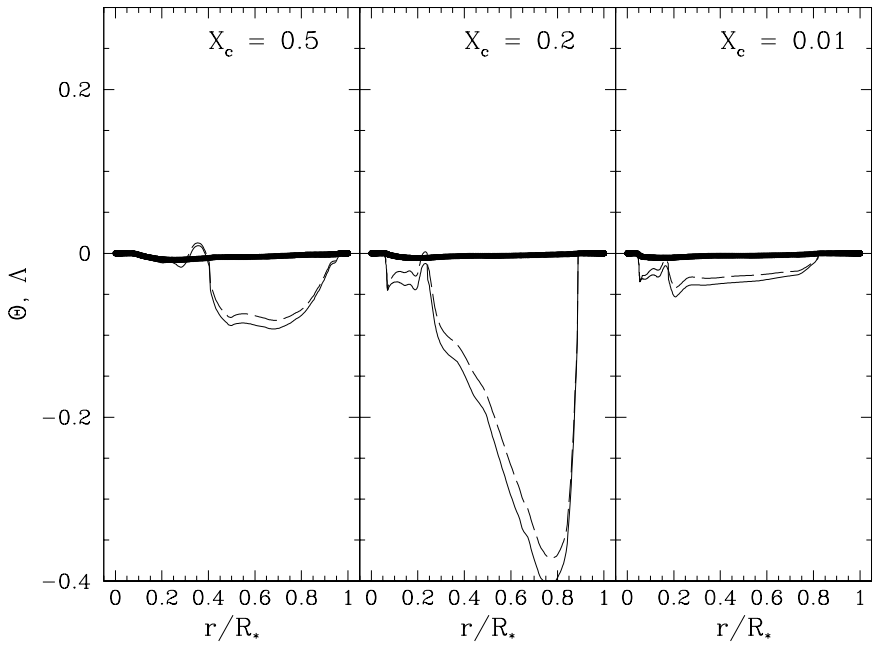

Fig. 4. Profiles of $\Theta$ and $\Lambda$ inside the $1.5 M_{\odot}$ star for three different values of the central hydrogen content. Solid lines are for $\Theta$, dashed ones for $\Lambda$. Thin lines stand for models where "complete mixing" $\left(E_{\mu} \neq 0\right)$ is applied while the bold line gives the profile of $\Theta$ when $E_{\mu}=0$.

the equation of state (Eq. (5)). Observations and theory show us that the circulation in main sequence stars on the hot side of the Li dip must act to extract angular momentum. In this configuration, the term $E_{\Omega}$ creates an anti-clockwise circulation transporting matter from the pole down to the equator. $E_{\mu}$, on the otherside, opposing $E_{\Omega}$ (see Eq. (3)), generates a clockwise circulation. The net effect is a reduction of angular momentum transfer to the surface and an enhancement of differential rotation.
In Fig. 4 we present profiles of $\Theta$ and $\Lambda$ at given evolutionary points. The bold line represents the $\Theta$ profile in the absence of $\mu$-currents. The difference between both is striking. It shows, as was already pointed out by Talon et al. (1997) in their study of a $9 M_{\odot}$ star, that the description of the rotation profile and of the circulation is not anymore a matter of $\Theta$ (or $\Omega$ ) alone, but of $\Theta-\Lambda$. Indeed, $\Theta$ is then conditioned by $\Lambda$ due again to their mirrored evolution (Eqs. (5) and (7)).

The large central differential rotation is a key ingredient in core evolution. Indeed, $\mu$-gradients in that region inhibit the development of a large turbulent diffusivity (see Eq. (13)). However, the large shear resulting from the mirror evolution of $\Theta$ and $\Lambda$ significantly increases local core mixing ${ }^{3}$.

Let us mention the work of Théado \& Vauclair (2001), who performed calculations including the effects of $\mu$-currents according to Vauclair (1999) in order to reproduce the low dispersion found in the "Spite lithium plateau". Assuming slow, constant, solid body rotation (a few $\mathrm{km} \mathrm{s}^{-1}$ in typical halo dwarfs), and that the transport of chemicals is progressively blocked when $E_{\mu}=E_{\Omega}$ (a situation they call creeping paralysis), they achieve a dispersion of 0.1 dex in the lithium plateau, in agreement with observations. We point out the fact that their scenario implies solid body rotation and thus prevents the rotation profile to react to horizontal molecular weight gradients as is the

\footnotetext{
${ }^{3}$ As explained in Talon et al. (1997), in a non-evolving model, Eq. (2) has a stationnary solution in which advection of momentum by the circulation is compensated by turbulent transport. There is thus a $\Theta$ equilibrium profile. When adding $\mu$-currents, about the same equilibrium exists for $\Theta-\Lambda$. This leads to $\Theta$ following the behavior of $\Lambda$, resulting in large differential rotation in the core.
} 


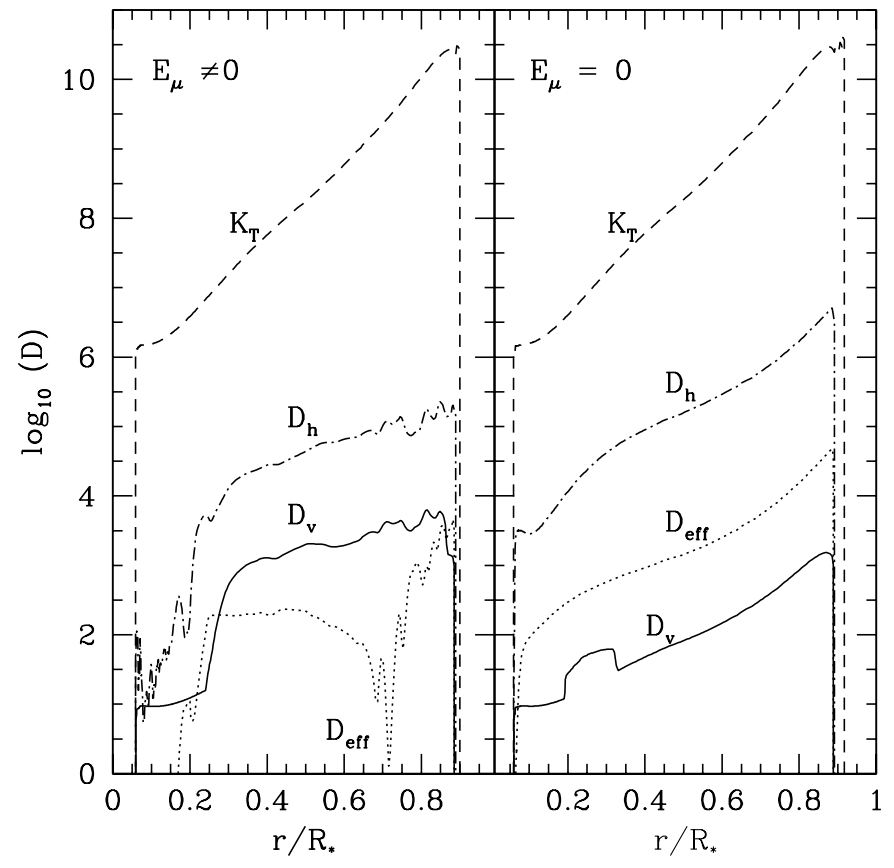

Fig. 5. Profiles of the different components of the diffusion coefficient and of the thermal diffusivity as a function of the reduced radius when $E_{\mu} \neq 0$ (left) and $E_{\mu}=0$ (right). The models correspond to the $1.5 M_{\odot}$ star with $X_{\mathrm{c}}=0.2$.

case in our description. Solid body rotation would be enforced in the presence of a small magnetic field or by gravity waves (Talon \& Charbonnel, in preparation), which might be the case in halo stars as well as in the stars lying on the cool side of the Li dip. However, as discussed by TC98, such an additional process of extraction of angular momentum is not expected to be efficient in the more massive stars lying on the left side of the Li dip which are under the scope of this study.

\subsection{Diffusion coefficients}

The examination of the total diffusion coefficients (from Fig. 2), reveals that, except towards the centre, they appear to be very much the same, whether $E_{\mu}$ is zero or not. The transport of chemicals, contrary to that of angular momentum, will thus not be significantly affected during main sequence evolution (see next section). However, while the total coefficients are of similar amplitude, they are not the result of the same combination of effects. Figure 5 presents various diffusion coefficients in both cases for a central hydrogen content $X_{c}=0.2$. We can firstly emphasize the fact that the shellular rotation is actually realistic, for $D_{\mathrm{h}} \gg D_{\mathrm{eff}}$ and $D_{\mathrm{v}}$. Secondly, when $E_{\mu} \neq 0$, the turbulent diffusion coefficient $D_{\mathrm{v}}$ is larger than the effective coefficient $D_{\text {eff }}$ almost everywhere in the radiative zone. This is a consequence of the enhancement of the differential rotation rate in this case, which favours the growth of turbulence ${ }^{4}$. Due to the strong anisotropy of turbulent motions, the buoyancy restoring forces in the vertical direction are slightly reduced because of the horizontal diffusion, and vertical turbulent

\footnotetext{
4 This appears clearly when turning to Eq. (17), through which $D_{\mathrm{v}} \propto(\mathrm{d} \Omega / \mathrm{d} r)^{2}$.
}

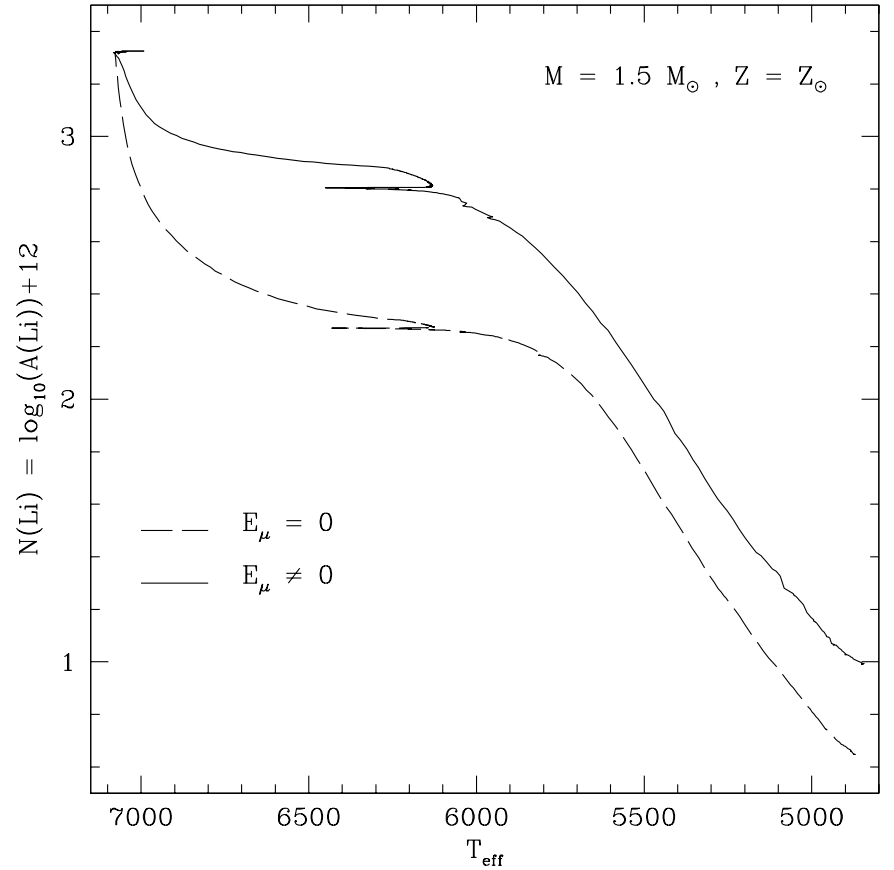

Fig. 6. Evolution of the surface abundance of lithium with temperature in the $1.5 M_{\odot}$ star for the two different approaches of mixing as mentioned on the plot and in the text.

mixing is allowed. The profile of the effective diffusion coefficient is influenced by $U(r)$ (Eq. (10)), so that it reflects the loops of the circulation in the presence of $\mu$-currents, each "drop" corresponding to an inversion of the direction of the flow.

When neglecting variations of $\mu$, turbulence can only marginally develop in a small portion of the radiative zone and the vertical diffusion coefficient $D_{\mathrm{v}}$ is reduced to molecular viscosity which is always smaller than $D_{\text {eff }}$.

In the central part, we may notice that the total diffusion coefficient is lowered by one order of magnitude in the presence of $\mu$-currents. As pointed out before, $\mu$-gradients in that region inhibit the development of an efficient turbulent diffusivity. This appears clearly in Fig. 5 (left panel), where we see $D_{\text {eff }}$ dropping down below $10^{1}$ just above the core. $D_{\mathrm{v}}$ also decreases in the same region, where contrary to the rest of the radiative zone, it is dominated by the molecular viscosity rather than by the turbulent viscosity (there is no turbulence).

Figure 6 presents a comparison between the evolution of the lithium surface abundance in the $1.5 M_{\odot}$ rotating star when considering or not the effects of $\mu$-currents. Both tracks are similar, but the depletion of lithium during the main sequence is larger in case of $E_{\mu}=0$. The surface abundance of lithium differs by more than 0.3 dex at 700 Myrs, which is more than the observational errorbar commonly assumed. Accounting for $\mu$ variations reduces the effect of mixing when all others assumptions remain the same. We have seen that $\mu$-currents rule the evolution of the rotation profile. They lead to an enhancement of the degree of differential rotation, which in turn allows for the horizontal turbulence to develop more easily. This horizontal turbulence hinders the transport towards the vertical direction. This effect shows up through the surface abundance of 


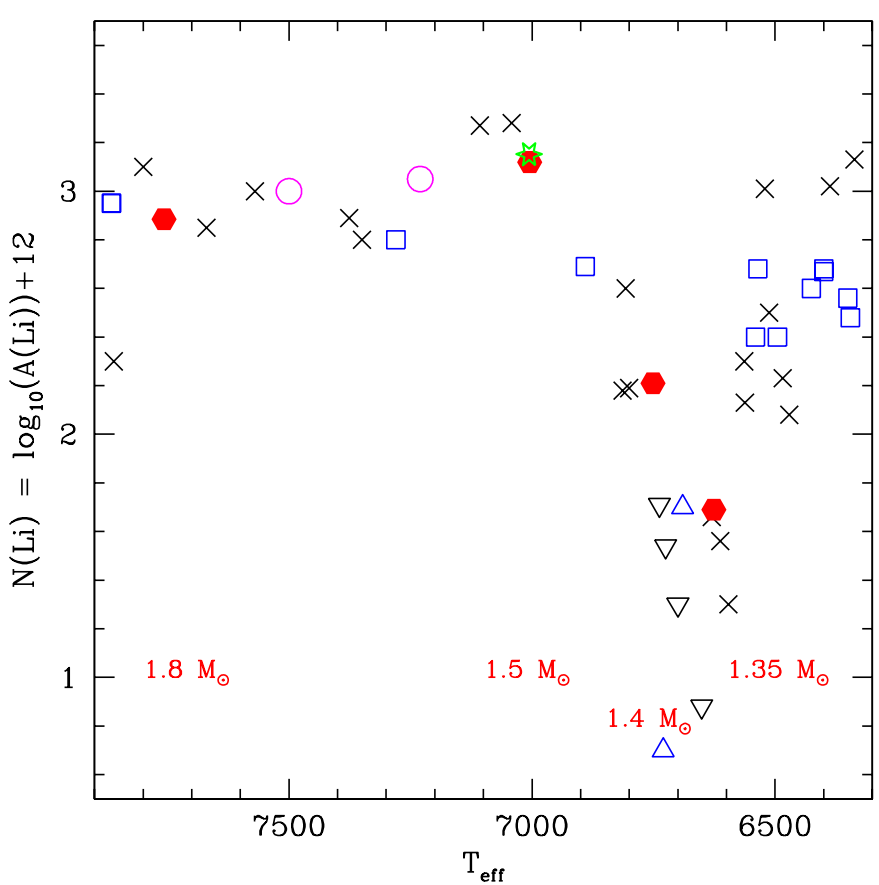

Fig. 7. Comparison of the models (hexagons and open bold star) with observations in the Hyades (crosses), in Praesepe (squares) and in Coma Berenices (circles and triangles). Downward triangles are for upper limits. Observations are from Burkhart \& Coupry $(1998,2000)$ and Boesgaard (1987). The corresponding masses of the models are as indicated on the figure. All models presented have $Z=0.02$ and an initial velocity of $110 \mathrm{~km} \mathrm{~s}^{-1}$; the reader is referred to Table 2 for details on braking. For the $1.5 M_{\odot}$ model, open bold star is obtained when not including microscopic diffusion on $\mathrm{LiBeB}$ while for hexagons microscopic diffusion was included also for these light elements (see text for further details).

lithium, that is less depleted when $E_{\mu} \neq 0$. Nevertheless, this abundance feature can not be advocated as an observational clue to the action of the $\mu$-currents. Indeed, braking plays a major role in determining the amplitude of mixing (and thus the amplitude of lithium depletion), and thus may rub off the effects of the variations of $\mu$. The surface velocity reached for $T_{\text {eff }} \simeq 4900 \mathrm{~K}$ is of a few $\mathrm{km} \mathrm{s}^{-1}$ in both cases, velocity for the model with $E_{\mu}=0$ being slightly larger $\left(9 \mathrm{~km} \mathrm{~s}^{-1}\right.$ versus $6.5 \mathrm{~km} \mathrm{~s}^{-1}$ when $E_{\mu} \neq 0$ ), because of the different braking constant used in this case in order to achieve the same surface velocity at $700 \mathrm{Myrs}$ as in absence of $\mu$-currents. Both velocities reached at the end of the computation are consistent with observations (Gaigé 1993). As a result, accounting for the effects of $\mu$-currents under the assumptions described in Sect. 3 does not make any significant change in the outcoming surface abundances compared to the case without variations of $\mu$. The observed abundances used as constraints do not allow to distinguish between these two cases. However we pointed out differences in the final rotation profiles, which might change significantly rotational transport during later evolutionary phases.

\section{Comparison with observations}

We now compare the predictions of our rotating-models with the observations of $\mathrm{Li}, \mathrm{Be}$ and $\mathrm{CNO}$ on the hot side of the

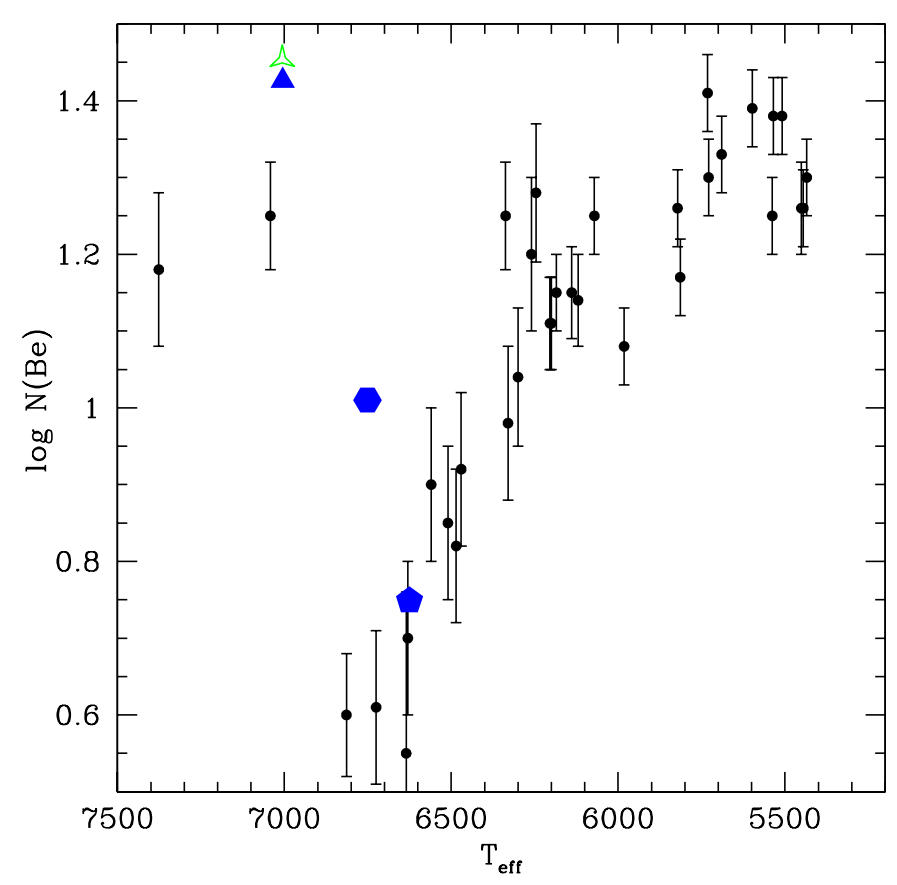

Fig. 8. Beryllium abundance as a function of effective temperature in the Hyades (Boesgaard \& King 2002). The pentagon, hexagon and triangle are the values obtained for the $1.35 M_{\odot}, 1.4 M_{\odot}$ and $1.5 M_{\odot}$ models respectively. The empty triangle is a $1.5 M_{\odot}$ model where microscopic diffusion is not applied to LiBeB. Refer to Table 1 for further details on braking.

so-called Li dip at the age of the Hyades, and of Li in subgiants of solar metallicity.

\section{1. $\mathrm{Li}, \mathrm{Be}$ and CNO on the hot side of the Li dip in the open clusters}

The drop-off in the Li content of main-sequence $F$ stars in a narrow range of effective temperature centred around $6700 \mathrm{~K}$, or "Li dip" (Wallerstein et al. 1965; Boesgaard \& Tripicco 1986; Balachandran 1995), is the most striking signature of transport processes that occur inside these stars. We refer to TC98 and CT99 for a detailed discussion of the different processes which have been proposed to account for this feature. These papers show how rotation induced mixing shapes in a very natural way the hot side of the Li dip. As already mentioned, we did not consider the effects of the $\mu$ gradients in these previous papers. However, as discussed in Sect. 4.2.3, the total diffusion coefficients, and thus the resulting transport of chemicals, is not significantly affected by these terms during main sequence. As a consequence, the comparison of the predictions of $\mathrm{Li}$ and $\mathrm{Be}$ surface abundances with observations on the hot side of the Li dip remains highly satisfactory, as can be seen in Figs. 7 and 8.

In CT99, microscopic diffusion on LiBeB had been turned off (while it was applied to all other chemicals) for stars with $T_{\text {eff }}$ higher than $6900 \mathrm{~K}$, because of the competition between radiative acceleration and gravity (Richer \& Michaud 1993; Richer et al. 2000, see discussion Sect. 3.4). In Figs. 7 and 8 , we present the results of computations with and without gravitational settling of $\mathrm{LiBeB}$ at the effective temperature 


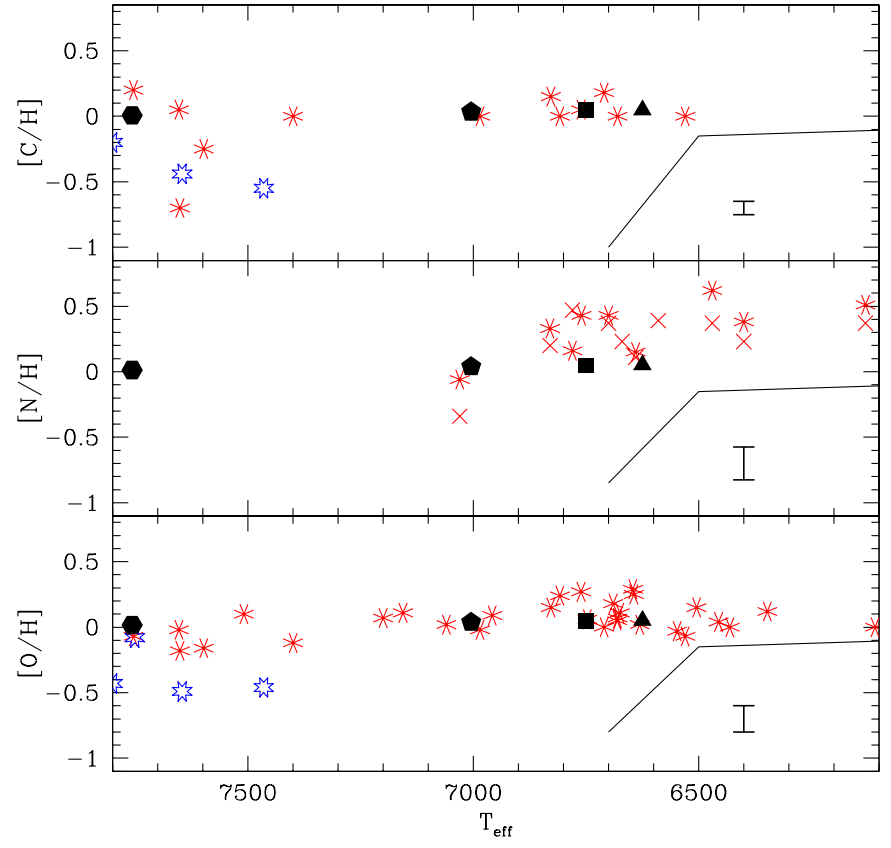

Fig. 9. Abundances of carbon, nitrogen and oxygen vs effective temperature in the Hyades, presented as asterisks and open stars for A and Am stars respectively (the latter ones are not meant to be reproduced here; see text for details). Observations of $\mathrm{C}$ and $\mathrm{O}$ are from Varenne \& Monier (1998). Observations of $N$ are from Takeda et al. (1998), crosses are derived from the $8629 \AA$ line and stars are from $8683 \AA$ line. Abundances are given relative to solar values from Grevesse \& Noels (1993). The typical errorbar for observational values is given for each panel. Models with complete mixing, a turbulence parameter $\mathrm{C}_{\mathrm{h}}=1$ and braking as reported in Table 2 are represented by the filled points with hexagon, pentagon, square and triangle standing for 1.8, $1.5,1.4$ and $1.35 M_{\odot}$ respectively, with $Z_{\star}=0.02$. Lines are from calculations including gravitational settling and radiative forces by Turcotte et al. (1998) (see Varenne \& Monier 1999 for further details).

where radiative forces can over-compensate gravitation. As can be seen, for the rotationnal velocities considered here, the differences are minute. In addition, the effect on stellar structure itself is null, since $\mathrm{LiBeB}$ are trace elements.

Observations of $\mathrm{C}, \mathrm{N}$ and $\mathrm{O}$ are precious clues on transport mechanisms inside main-sequence F-type stars, and on the competition between atomic diffusion and macroscopic processes. Indeed, in the case of pure atomic diffusion, these elements are expected to be underabundant in the $\mathrm{Li} \mathrm{dip}$, as shown in Fig. 9. On the other hand, in our models rotation induced mixing strongly inhibits the settling effects. As a result, the surface abundances of CNO do not vary in the $T_{\text {eff }}$ range considered. This is in perfect agreement with observations in the Hyades (Varenne \& Monier 1998; Takeda 1998; see also Boesgaard 1989; Friel \& Boesgaard 1990; Garcia Lopez et al. 1993), as can be seen in Fig. 9. Let us stress that in the temperature domain of A-type stars, most of the observations report abundances for chemically peculiar objects, namely Am stars, that are slow rotators in which the action of gravitational settling is important on CNO elements. These peculiar patterns might be achieved by considering the combined effects of large scale circulation, turbulence, gravitational settling and radiative forces (the effects of the latter not being included in our evolutionary code) in models with a slow initial rotation. Such computations are not presented here for this is out of the scope of the present study to focus on such a particular phenomenon.

\subsection{Li in subgiants}

Early observations by Alschuler (1975) of a few field stars crossing for the first time the Hertzsprung gap indicated that lithium depletion starts earlier than predicted by standard dilution for stars more massive than $2 M_{\odot}$. Later on, significant lithium depletion was observed in a non negligible number of slightly evolved field stars (Brown et al. 1989; Balachandran 1990). Do Nascimento et al. (2000) studied the behaviour of lithium in a sample of field subgiants observed by Lèbre et al. (1999) for which Hipparcos data allowed the precise determination of both evolutionary status and mass. They confirmed that stars originating from the hot side of the dip present a large range of lithium abundances which can not be explained by standard dilution alone and which reflect different degrees of depletion of this element while on the main sequence, even if its signature does not appear at the stellar surface at the age of the Hyades (see Vauclair 1991; Charbonnel \& Vauclair 1992). In this cluster, dilution is not sufficient to explain lithium values in evolved stars (which have masses of the order of $2.2 M_{\odot}$ ), while main sequence stars present galactic abundance (Boesgaard et al. 1977; Duncan et al. 1998). This has been observed also in open clusters with turnoff masses higher than $\sim 1.6 M_{\odot}$ (Gilroy 1989; Pasquini et al. 2001).

In Fig. 10 we compare our predictions for the surface Li abundance of various models with the observations in both field and open cluster evolved stars. First note that in classical models without rotation the variation in surface $\mathrm{Li}$ abundance is very similar for stars of different masses because it is only due to the first dredge-up dilution. More importantly, classical Li depletion starts at a too low $T_{\text {eff }}$ (around $\sim 5850 \mathrm{~K}$ ) and leads to final $\mathrm{Li}$ abundances too high when compared to observational data. On the other hand, and even if no important lithium depletion is expected at the surface of the main sequence stars on the hot side of the dip at the age of the Hyades, more lithium destruction occurs inside rotating models compared to classical ones. Indeed, due to the enlargement of the Li free radiative regions, important surface Li depletion occurs at much higher $T_{\text {eff }}$ than in classical models. Also, total Li depletion after dredge-up is more important in rotating models than in models without transport processes. The predicted evolution of the surface lithium abundance in our rotating models explains the behaviour and the dispersion in field and open cluster evolved stars more massive than $\sim 1.4 M_{\odot}$.

\section{Conclusions}

To study the evolution of low mass rotating stars and the effects of rotation induced mixing on surface abundance patterns, we have presented here a first series of results concerning main sequence and subgiant Pop I stars located on the hot side of the lithium dip. We performed calculations including the treatment 


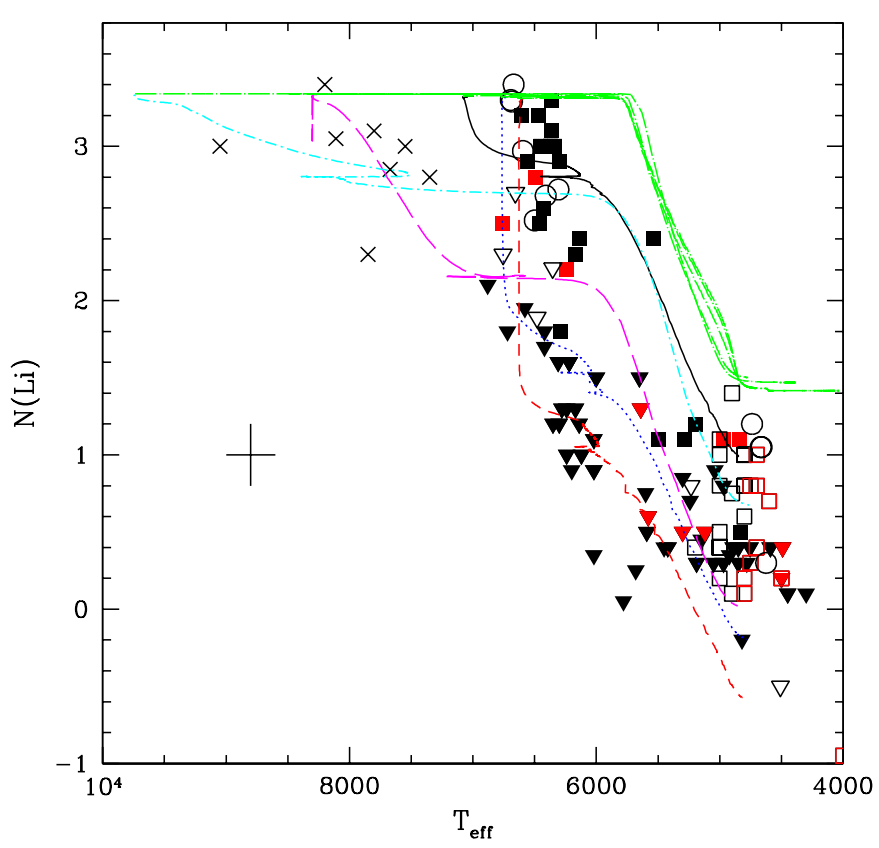

Fig. 10. Lithium abundance versus effective temperature in open clusters (open symbols) and field (filled symbols) subgiants compared with our rotating models. Triangles indicate upper limits. Observations are from Lèbre et al. (1999) (field), Wallerstein et al. (1994) (field), Pasquini et al. (2001) (NGC 3680), Gilroy (1989) (open clusters) and Burkhart \& Coupry $(1989,2000)$ (Hyades among other open clusters). Dot-dashed lines represent results for standard models of $1.35 M_{\odot}, 1.4 M_{\odot}, 1.5 M_{\odot}, 1.8 M_{\odot}$ and 2.2 $M_{\odot}$. They all coalesce in a single line, standard dilution starting around $5750 \mathrm{~K}$. Other lines are for models with rotation induced mixing and magnetic braking. Solid line, dotted line, long dashed line, dashed line and dot-dashed line are for $1.5 M_{\odot}, 1.4 M_{\odot}, 1.8 M_{\odot}, 1.35 M_{\odot}, 2.2 M_{\odot}$ respectively. Refer to Tab. 1 for details concerning rotational velocities.

of transport of angular momentum and chemical species due to meridional circulation, shear turbulence and gravitational settling according to Maeder \& Zahn (1998) formalism. In particular, we take into account horizontal variations of mean molecular weight, and their action on the transport itself. These terms prove to be very important in defining the shape of the internal rotation profile. Indeed the horizontal variations of $\mu$ and the derivative of $\Omega$, namely, $\Lambda$ and $\Theta$ respectively, have a mirrored behaviour, $\Theta$ following $\Lambda$ when it is not null. As soon as the circulation is settled, the $\mu$-currents grow larger and induce a stronger differential rotation.

On the other hand, the effects of these terms on the surface abundances of light elements appear not to be constraining compared with braking for instance. The major change introduced in the transport of chemicals when taking $\mu$-currents into account concerns the dominant contributor to the diffusion coefficient. In an "homogeneous" star (where there are no horizontal variations of $\mu$ ), the effective diffusion coefficient $D_{\text {eff }}$ is the major contributor, while in the inhomogeneous case, turbulent diffusion coefficient dominates the transport of chemicals.

Finally, contrary to what has been suggested in previous work concerning rotation induced mixing in solid body rotating low mass stars (typically halo main sequence stars with $M_{\star}<$ $0.9 M_{\odot}$ ), when considering extraction of angular momentum through magnetic braking, the situation of "creeping paralysis" of the circulation is not reached in the models presented here. It shall actually never be the case as long as differential rotation is allowed to exist for the mirrored terms never compensate each other.

The point to be emphasized is the fact that these variations of mean molecular weight are not to be neglected when considering rotating objects. They modify the action of mixing and can be of major importance after main sequence evolution, for they constrain the internal rotation profile.

Acknowledgements. We are grateful to the referee Pr. André Maeder and Pr. Sylvie Vauclair for their usefull comments, that helped to improve this paper. We wish to thank the french "Programme National de Physique Stellaire" for financial support. S.T. was supported by NSERC of Canada and by the Canada Research Chair in Stellar Astrophysics awarded to G. Fontaine.

\section{References}

Alexander, D. R., \& Ferguson, J. W. 1994, ApJ, 437, 879

Alschuler, D. R. 1975, ApJ, 195, 649

Angulo, C., Arnould, M., Rayet, M., et al. 1999, Nucl. Phys. A, 656, 3

Balachandran, S. 1990, ApJ, 354, 310

Balachandran, S. 1995, ApJ, 446, 203

Barnes, S., \& Sofia, S. 1996, ApJ, 462, 746

Boesgaard, A. M., Heacox, W. D., \& Conti, P. S. 1977, ApJ, 214, 124

Boesgaard, A. M., \& Tripicco, M. J. 1986, ApJ, 303, 724

Boesgaard, A. M. 1989, ApJ, 336, 798

Boesgaard, A. M., \& King, J. R. 2002, ApJ, 565, 587

Bouvier, J., Forestini, M., \& Allain, S. 1997, A\&A, 326, 1023

Brown, J. A., Sneden, C., Lambert, D. A., \& Dutchover, E. 1989, ApJS 71, 293

Burkhart, C., \& Coupry, M. F. 1989, A\&A, 220, 197

Burkhart, C., \& Coupry, M. F. 2000, A\&A, 354, 216

Canuto, V. M. 1998, ApJ, 505, L47

Chaboyer, B., Demarque, P., Guenther, D. B., \& Pinsonneault, M. H. 1995, ApJ, 446, 435

Chaboyer, B., \& Zahn, J. P. 1992, A\&A, 253, 173

Charbonnel, C., \& Vauclair, S. 1992, A\&A, 265, 55

Charbonnel, C., \& Talon, S. 1999, A\&A, 351, 635

do Nascimento, J. D., Charbonnel, C., Lèbre, A., de Laverny, P., \& de Medeiros, J. R. 2000, A\&A, 357, 931

Duncan, D. K., Peterson, R. C., Thorburn, J. A., \& Pinsonneault, M. H. 1998, ApJ, 499, 871

Eddington, A. S. 1925, The Observatory, 48, 73

Friel, E. D., \& Boesgaard, A. M. 1990, ApJ, 351, 480

Gaigé, Y. 1993, A\&A, 269, 267

García, Lopez, R. J., Rebolo, R., Herrero, A., \& Beckman, J. E. 1993, ApJ, 412, 173

Gilroy, K. K. 1989, ApJ, 347, 835

Grevesse, N., \& Noels, A. 1993, Phys. Scr. T, 47, 133

Iglesias, C. A., \& Rogers, F. J. ApJ, 464, 943

Kawaler, S. D. 1988, ApJ, 333, 236

Kim, Y. C., \& Demarque, P. 1996, ApJ, 457, 340

Kippenhahn, R. 1974, Late Stages of Stellar Evolution, IAU Symp., 66,20

Krishnamurthi, A., Pinsonneault, M. H., Barnes, S., \& Sofia, S. 1997, ApJ, 480, 303

Lèbre, A., de Laverny, P., de Medeiros, J. R., Charbonnel, C., \& da Silva, L. 1999, A\&A, 345, 936 
Lebreton, Y. 2000, ARA\&A, 38, 35

Maeder, A., \& Zahn, J. P. 1998, A\&A, 334, 1000

Matias, J., \& Zahn, J. P. 1997, Sounding solar and stellar interiors, IAU Symp. 181, Nice, ed. J. Provost, \& F. X. Schmider

Mestel, L. 1953, MNRAS, 113, 716

Meynet, G., Mermilliod, J.-C., \& Maeder, A. 1993, A\&AS, 98, 477

Meynet, G., \& Maeder, A. 2000, A\&A, 361, 101

Moss, D. 1979, A\&A, 78, 119

Palacios, A., Charbonnel, C., Forestini, M., \& Talon, S. 2002, A\&A, in preparation

Paquette, C., Pelletier, C., Fontaine, G., \& Michaud, G. 1986, ApJS, 61,177

Pasquini, L., Randich, S., \& Pallavicini, R. 2001, A\&A, 374, 1017

Peréz Hernández, F., Claret, A., Hernández, M. M., \& Michel, E. 1999, A\&A, 346, 586

Pols, O. R., Tout, C. A., Eggleton, P. P., \& Han, Z. 1995, MNRAS, 274, 964

Richer, J., \& Michaud, G. 1993, ApJ, 416, 312

Richer, J., Michaud, G., \& Turcotte, S. 2000, ApJ, 529, 338

Saar, S. H. 1996, in Stellar surface structure: Proc. of the 176th Symp. of the International Astronomical Union, ed. K. G. Strassmeier, \& J. L. Linsky

Schatzman, E. 1962, Ann. Ap., 25, 18

Sills, A., \& Pinsonneault, M. 2000, ApJ, 540, 489

Siess, L., Forestini, M., \& Bertout, C. 1997, A\&A, 326, 1001
Siess, L., Dufour, E., \& Forestini, M. 2000, A\&A, 358, 593

Stauffer, J. R., \& Hartmann, L. W. 1987, ApJ, 318, 337

Takeda, Y., Kawanomoto, S., Takada-Hidai, M., \& Sadakane, K. 1998, PASJ, 50, 509

Talon, S., \& Charbonnel, C. 1998, A\&A, 335, 959

Talon, S., \& Charbonnel, C. 2003, A\&A, in preparation

Talon, S., \& Zahn, J. P. 1997, A\&A, 317, 749

Talon, S., \& Zahn, J. P., Maeder, A., \& Meynet, G. 1997, A\&A, 322, 209

Tassoul, J. L., \& Tassoul, M. 1982, ApJS, 49, 317

Théado, S., \& Vauclair, S. 2001, A\&A, 375, 70

Townsend, A. A. 1958, J. Fuid. Mech., 4, 361

Turcotte, S., Richer, J., Michaud, G., \& Christensen-Dalsgaard, J. 2000, A\&A, 360, 603

Varenne, O., \& Monier, R. 1999, A\&A, 351, 247

Vauclair S., in Evolution of stars: the photospheric abundance connection, ed. G. Michaud, \& A. Tutukov, IAU Symp., 145, 327

Vauclair, S. 1999, A\&A, 351, 973

Vauclair, G. 1979, A\&A, 55, 147

Vogt, H. 1926, Astron. Nachrichten, 227, 325

Wallerstein, G., Herbig, G. H., \& Conti, P. S. 1965, ApJ, 141, 610

Wallerstein, G., Böhm-Vitense, E., Vanture, A. D., \& Gonzalez, G. 1994, AJ, 107, 221

Zahn, J. P. 1992, A\&A, 265, 115

Zahn, J. P., Talon, S., \& Matias, J. 1997, A\&A, 322, 320 